\title{
Competencias Médicas
}

\author{
José María Peinado Herreros ${ }^{1}$ \\ Decano de la Facultad de Medicina de la Universidad de Granada
}

Entendemos por competencias el conjunto de conocimientos, habilidades y actitudes que permiten una excelente práctica médica, en continuo perfeccionamiento, adecuada al contexto social en que se desarrolla.

Uno de los elementos dinamizadores del debate sobre la formación en competencias es el proceso de convergencia europeo, el cual, junto a aspectos cognitivos (conocer y comprender), presta mayor atención a las habilidades psicomotoras (saber como actuar) y a la formación en valores y actitudes (saber como ser). Además centra el proceso educativo en el aprendizaje del alumno, mas que en la enseñanza, haciendo énfasis en los resultados finales.

Junto a la convergencia europea se han producido en nuestro país otros cambios normativos relevantes en la formación universitaria, entre los que destaca la Ley Orgánica de Universidades, (LOU), que ha introducido, entre otros aspectos, cambios en la carrera académica, y la Ley de Ordenación de las Profesiones Sanitarias (LOPS) que propone como novedad en el acceso a la formación especializada, examen MIR, la evaluación de los "conocimientos teóricos y prácticos y las habilidades clínicas y comunicativas". Finalmente no podemos olvidar la reciente actualización de la directiva europea relativa al "Reconocimiento de Cualificaciones Profesionales (Directiva europea 2021 (1). 12 2004,13781/2/04 REV 2, DE 21 de Diciembre de 2004)" (2), que considera la "Formación básica del médico" (al menos 6 años de estudios o 5500 horas de enseñanza teórica y práctica), y las "Condiciones Minimas de Formación (Conocimientos y Competencias)".

En este contexto, la Conferencia Nacional de Decanos de Facultades de Medicina Españolas, tomando como referencia la nueva normativa sobre convergencia Europea, e inspirándose en los requisitos globales mínimos esenciales en Educación Médica

1. El presente documento se basa en el "Libro Blanco" de la titulación de Medicina, ANECA, 2005, elaborado por la Conferencia Nacional de Decanos de Facultades de Medicina Españolas, siendo sus decanos los que se relacionan al final de este trabajo. Borrador disponible en www.aneca.es/librosblancos

2. Las competencias denominadas genéricas o transversales han sido tomadas del Proyecto Tuning. del Instituto Internacional para la Educación Médica, ha propuesto las competencias específicas ${ }^{2}$ que deben aprenderse y dominarse al concluir la formación básica del médico. Estas se ha clasificado en:

\section{a. VALORES PROFESIONALES, ACTITUDES, COMPORTAMIENTOS Y ÉTICA}

1. Reconocer los elementos esenciales de la profesión médica, incluyendo los principios éticos y las responsabilidades legales.

2. Comprender la importancia de tales principios para el beneficio del paciente, de la sociedad y la profesión, con especial atención al secreto profesional

3. Saber aplicar el principio de justicia social a la práctica profesional.

4. Desarrollar la práctica profesional con respeto a la autonomía del paciente, a sus creencias y cultura.

5 . Reconocer las propias limitaciones y la necesidad de mantener y actualizar su competencia profesional.

6. Desarrollar la práctica profesional con respeto a otros profesionales de la salud.

\section{b. FUNDAMENTOS CIENTÍFICOS DE LA MEDICINA}

7. Comprender y reconocer la estructura y función normal del cuerpo humano, a nivel molecular, celular, tisular, orgánico y de sistemas, en las distintas etapas de la vida.

8. Reconocer las bases de la conducta humana normal y sus alteraciones.

9. Comprender y reconocer los efectos, mecanismos y manifestaciones de la enfermedad sobre la estructura y función del cuerpo humano.

10. Comprender y reconocer los agentes causantes y factores de riesgo que determinan los estados de salud y el desarrollo de la enfermedad.

11. Comprender y reconocer los efectos del crecimiento, el desarrollo y el envejecimiento sobre el individuo y su entorno social.

12. Comprender los fundamentos de acción, indicaciones y eficacia de las intervenciones terapéuticas, basándose en la evidencia científica disponible 


\section{c. HABILIDADES CLÍNICAS}

13. Obtener y elaborar una historia clínica que contenga toda la información relevante.

14. Realizar un examen físico y una valoración mental.

15. Tener capacidad para elaborar un juicio diagnóstico inicial y establecer una estrategia diagnóstica razonada.

16. Reconocer y tratar las situaciones que ponen la vida en peligro inmediato, y aquellas otras que exigen atención inmediata.

17. Establecer el diagnóstico, pronóstico y tratamiento aplicando los principios basados en la mejor información posible.

18. Indicar la terapéutica más adecuada de los procesos agudos y crónicos más prevalentes, así como de los enfermos en fase terminal.

19. Plantear y proponer las medidas preventivas adecuadas a cada situación clínica.

\section{d. HABILIDADES DE COMUNICACIÓN}

20. Escuchar con atención, obtener y sintetizar información pertinente acerca de los problemas que aquejan al enfermo, y comprender el contenido de esta información.

21. Redactar historias clínicas y otros registros médicos de forma comprensible a terceros.

22. Comunicarse de modo efectivo y claro, tanto de forma oral como escrita con los pacientes, los familiares, los medios de comunicación y otros profesionales.

23. Establecer una buena comunicación interpersonal, que capacite para dirigirse con eficiencia y empatía a los pacientes, a los familiares, medios de comunicación y otros profesionales.

\section{e. SALUD PÚBLICA Y SISTEMAS DE SALUD}

24. Reconocer los determinantes de la salud en la población, tanto los genéticos como los dependientes del estilo de vida, demográficos, ambientales, sociales, económicos, psicológicos y culturales.

25. Asumir su papel en las acciones de prevención y protección ante enfermedades, lesiones o accidentes y mantenimiento y promoción de la salud, tanto a nivel individual como comunitario.

26. Reconocer su papel en equipos multiprofesionales, asumiendo el liderazgo cuando sea apropiado, tanto para el suministro de cuidados de la salud, como en las intervenciones para la promoción de la salud.

27. Obtener y utilizar datos epidemiológicos y valorar tendencias y riesgos para la toma de decisiones sobre salud.

28. Conocer las organizaciones internacionales de salud, y los entornos y condicionantes de los diferentes sistemas de salud.

\section{f. MANEJO DE LA INFORMACIÓN}

29. Conocer, valorar críticamente y saber utilizar las fuentes de información clínica y biomédica para obtener, organizar, interpretar y comunicar la información científica y sanitaria.

30. Saber utilizar las tecnologías de la información y la comunicación en las actividades clínicas, terapéuticas, preventivas y de investigación.

31. Mantener y utilizar los registros con información del paciente para su posterior análisis, preservando la confidencialidad de los datos.

\section{g. ANÁLISIS CRÍTICO E INVESTIGACIÓN}

32. Tener, en la actividad profesional, un punto de vista crítico, creativo, con escepticismo constructivo y orientado a la investigación.

33. Comprender la importancia y las limitaciones del pensamiento científico en el estudio, la prevención y el manejo de las enfermedades.

34. Ser capaz de formular hipótesis, recolectar y valorar de forma crítica la información para la resolución de problemas, siguiendo el método científico.

Una vez definidas las competencias se han sometido a la consideración, mediante encuesta, de 5 colectivos de médicos: Profesores permanentes y contratados, residentes, gestores y profesionales sin vinculación con la universidad. Se le pedía al encuestado, que puntuara del 1 al 4, cada una de las competencias de cada bloque, siendo 1 poco importante y 4 muy importante.

Es de destacar que el promedio del valor concedido a la totalidad de las competencias es de 3,44 , un valor alto considerando que el máximo posible sería 4. Las competencias con un valor promedio mas elevado son "Reconocer y tratar las situaciones que ponen la vida en peligro inmediato, y aquellas otras que exigen atención inmediata" así como "Obtener y elaborar una historia clínica que contenga toda la información relevante", ambas dentro del bloque de habilidades clínicas. En tercer lugar en relevancia 
para todos los grupos encuestados se sitúa "escuchar con atención, obtener y sintetizar información pertinente acerca de los problemas que aquejan al enfermo, y comprender el contenido de esta información", dentro del bloque de habilidades de comunicación.

En el "Libro Blanco" se relacionan cada una de las competencias mencionadas con los bloques en los que se estructura la titulación. También este documento propone en el Anexo II el saber y saber hacer de la formación médica universitaria.

Universidades participantes en la elaboración del "Libro Blanco de la Titulación de Medicina", y Profesores responsables.

1. Universidad de Alcalá: Prof. José Vicente Saz Pérez. Decano.

2. Universidad Autónoma de Barcelona: Prof. Alvar Net Castel. Decano.

3. Universidad Autónoma de Madrid: Prof. Valentín Cuervas-Mons. Decano

4. Universidad de Barcelona: Profa. M ${ }^{\mathrm{a}}$ Teresa Estrach Panella. Decana.

5. Universidad de Cádiz: Prof. Antonio José Chover. Vicedecano.

6. Universidad de Cantabria: Prof. José Antonio Amado Señaris. Decano.

7. Universidad de Castilla-La Mancha: Prof. José Laborda Fernández. Decano. (Prof. José Juiz. Decano al inicio del proyecto).

8. Universidad Complutense de Madrid: Prof. Ángel Nogales Espert. Decano.

9. Universidad de Córdoba: Prof. Francisco Pérez Jiménez. Decano.

10. Universidad de Extremadura: Prof. Pedro Bureo Dacal. Decano. (Prof. Domingo Macías. Decano al inicio del proyecto).
11. Universidad de Granada: Prof. José $\mathrm{M}^{\mathrm{a}}$ Peinado Herreros. Decano.

12. Universidad de La Laguna: Prof. Eduardo Domenech. Decano. (Prof. Luis Hernández Nieto. Decano al inicio del proyecto).

13. Universidad de Las Palmas de Gran Canaria: Prof. Juan Cabrera Cabrera. Decano.

14. Universidad de Lleida: Prof. Ángel Rodríguez Pozo. Decano.

15. Universidad de Málaga: Prof. Salvador González Baron. Decano. (Prof. Ignacio Pérez de Varga. Decano al inicio del proyecto).

16. Universidad Miguel Hernández: Prof. Juan Caturla Such. Decano.

17. Universidad de Murcia: Prof. Fernando Sánchez Gascón. Decano. (Prof. Francisco Monserrat Bernal. Decano al inicio del proyecto).

18. Universidad de Navarra: Profa. Ma Pilar Civeira Murillo. Decana.

19. Universidad de Oviedo: Prof. Enrique Martínez Rodríguez. Decano.

20. Universidad del País Vasco: Prof. Agustín Martínez Ibargüen. Decano. (Profa. $\mathrm{M}^{\mathrm{a}}$ Luisa Ugedo. Decana al inicio del proyecto).

21. Universidad Rovira i Virgili: Prof. Rodrigo Miralles Marrero. Decano.

22. Universidad de Salamanca: Prof. José I. Paz Bouza. Decano.

23. Universidad de Santiago de Compostela: Prof. José $\mathrm{M}^{\mathrm{a}}$ Fraga Bermúdez. Decano.

24. Universidad de Sevilla: Profa. Carmen Osuna Fernández. Decana.

25. Universidad de Valencia: Prof. Esteban Morcillo Sánchez. Decano.

26. Universidad de Valladolid: Prof. Santiago Rodríguez. Decano.

27. Universidad de Zaragoza: Prof. Arturo Vera Gil. Decano. 